\title{
ARTICLE
}

\section{"K-effective of the World" and Other Concerns for Monte Carlo Eigenvalue Calculations}

\author{
Forrest B. BROWN
}

Los Alamos National Laboratory, Los Alamos, NM, 87545, USA

\begin{abstract}
Monte Carlo methods have been used to compute $k_{\text {eff }}$ and the fundamental mode eigenfunction of critical systems since the 1950s. Despite the sophistication of today's Monte Carlo codes for representing realistic geometry and physics interactions, correct results can be obtained in criticality problems only if users pay attention to source convergence in the Monte Carlo iterations and to running a sufficient number of neutron histories to adequately sample all significant regions of the problem. Recommended best practices for criticality calculations are reviewed and applied to several practical problems for nuclear reactors and criticality safety, including the "K-effective of the World" problem. Numerical results illustrate the concerns about convergence and bias. The general conclusion of is that with today's high-performance computers, improved understanding of the theory, new tools for diagnosing convergence (e.g., Shannon entropy of the fission distribution), and clear practical guidance for performing calculations, practitioners will have a greater degree of confidence than ever of obtaining correct results for Monte Carlo criticality calculations.
\end{abstract}

KEYWORDS: convergence, undersampling, bias, criticality

\section{Introduction}

Monte Carlo methods have been used to compute $k_{\text {eff }}$ and the fundamental mode eigenfunction of critical systems since the 1950s. During the 1960-70s, pioneering work by Lieberoth, ${ }^{1)}$ Gelbard and Prael, ${ }^{2)}$ and Brissendon and Garlick ${ }^{3)}$ examined the mathematical basis for performing criticality calculations using Monte Carlo methods. Three principle concerns were identified that must be addressed to perform calculations correctly: (1) Sufficient initial cycles must be discarded prior to beginning the tallies, so that contamination of the results by the initial source guess becomes negligible. (2) Sufficient numbers of neutrons must be followed in each cycle so that bias in $k_{\text {eff }}$ and reaction rate tallies becomes negligible. (3) Bias in the statistics on $k_{\text {eff }}$ and reaction rate tallies must be recognized and dealt with.

In 1971, Whitesides ${ }^{4)}$ took a more practical approach to the problem by defining the "K-effective of the World" problem:

$\ldots$ if one attempts to calculate the $k_{\text {eff }}$ of the world using a Monte Carlo calculation, what $\mathrm{k}_{\text {eff }}$ would be computed assuming that there are several critical assemblies located around the world? The answer would likely be the $k_{\text {eff }}$ of the world with no critical assemblies present. The cause of the erroneous result is the fact that the volume of fissile material in the world would be so large relative to the volume of fissile material in the critical assemblies that most commonly used forms of sampling would almost never "see" the critical assemblies. Hence, this would not reflect their existence in the computed $k_{\text {eff }}$.

..... The erroneous results for these types of problems are the result of the failure of the calculation to converge the source to the fundamental source mode.

*Corresponding author, E-mail: fbrown@lanl.gov

(C) 2011 Atomic Energy Society of Japan, All Rights Reserved.
Whitesides also defined a model problem to illustrate the potential problems from undersampling the fission source and not properly converging the fission source. More recently, the OECD/NEA Expert Group On Source Convergence in Criticality Safety Analysis ${ }^{5,6)}$ investigated source convergence issues for Monte Carlo calculations and suggested improvements to Monte Carlo codes and guidance for code users. ${ }^{5,6,7)}$

\section{Monte Carlo Criticality Calculations}

Most Monte Carlo codes use the standard power method for solving k-eigenvalue problems, ${ }^{7,8,9}$ where each (outer) iteration cycle corresponds to a single fission generation in the simulation. Given a fission neutron source distribution and an estimate of $k_{\text {eff, }}$, single-generation random walks are carried out for a "batch" of neutrons to estimate a new $k_{\text {eff }}$ and fission source distribution. Iterations continue until both $k_{\text {eff }}$ and the fission source distribution have converged. After convergence of the power iterations, tallies of $k_{\text {eff }}$ and spatial reaction rates are accumulated.

As in all Monte Carlo calculations, if there are too few neutrons in a cycle to adequately sample the problem phase space, results will not be accurate and statistics will be meaningless. Sufficient neutrons/cycle must be used to adequately cover the problem phase space. In addition, the Monte Carlo implementation of the power method suffers from a bias, ${ }^{2,3)}$ in that the normalization of the fission source after each cycle involves dividing by a stochastic quantity (i.e., all ratio estimators are biased). Fortunately, the renormalization bias is inversely proportional to the number of neutrons/cycle. Thus, using a large number of neutrons/cycle 
reduces or eliminates difficulties from both undersampling and renormalization bias.

The other principal concern for Monte Carlo criticality calculations is determining how many iteration cycles are required to converge the fission source distribution. Sufficient initial cycles must be discarded prior to beginning the tallies, so that contamination of the results by the initial source guess becomes negligible. Shannon entropy of the fission source distribution has been used recently to characterize the source convergence. ${ }^{8)}$

\section{Numerical Results}

Three sample problems were chosen to represent typical reactor and criticality safety calculations and illustrate the effects of undersampling, convergence, and bias: (1) the K-effective of the World problem specified by Whitesides,
(2) a 2D quarter-core Pressurized Water Reactor (PWR) model, and (3) an array of cans partially filled with plutonium nitrate solution. Criticality calculations for these 3 problems were performed using $\mathrm{MCNP}^{9,10)}$ with continuous-energy ENDF/B-VII data. The problems were run many different ways, including poorly (too few neutrons/cycle) and according to recommended best practices. ${ }^{7)}$ It should be noted that the theory and results presented below apply not just to MCNP5, but to any Monte Carlo codes that use the power iteration method to solve criticality problems.

\section{K-effective of the World Problem}

Figure 1 provides results from MCNP5 calculations of the model problem specified by Whitesides. ${ }^{4}$ Figure 1B shows the convergence behavior of $k_{e f f}$ and $H_{s r c}$, the Shannon
A

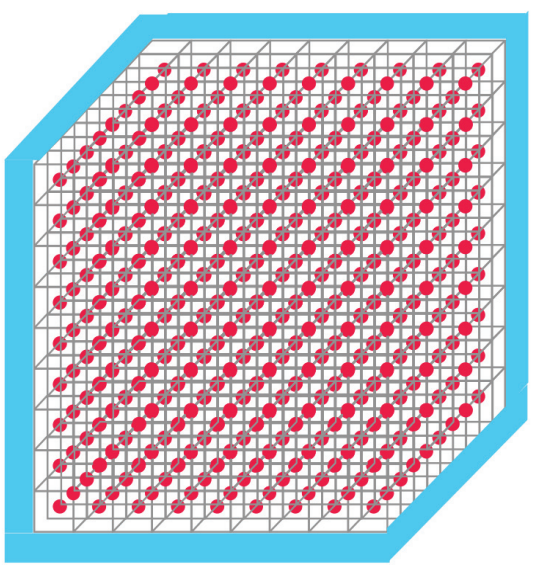

C

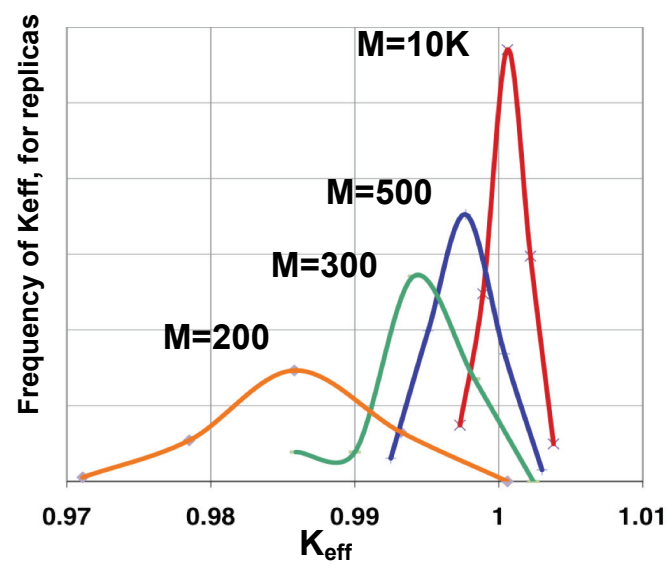

B

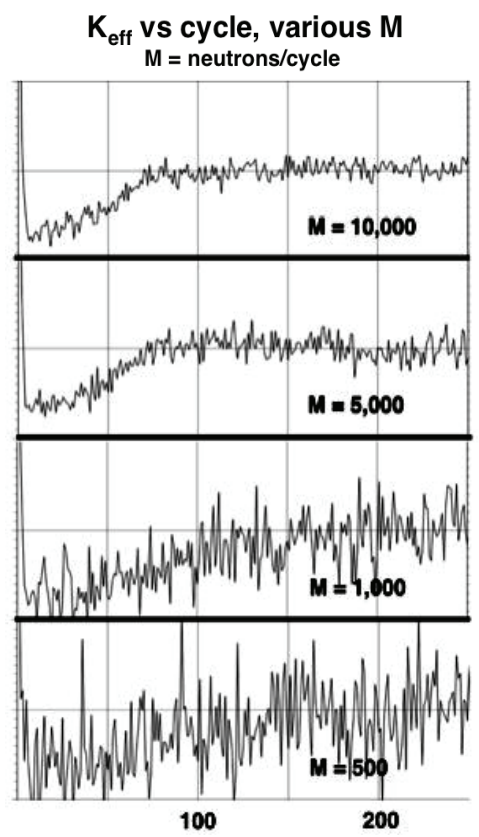

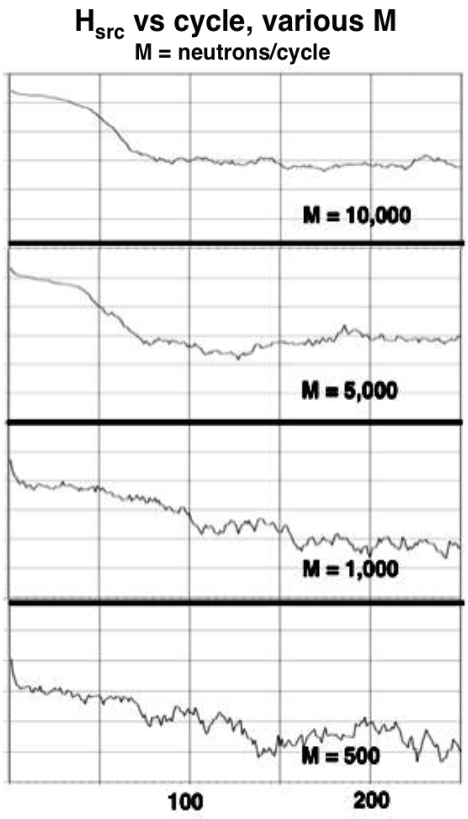

D

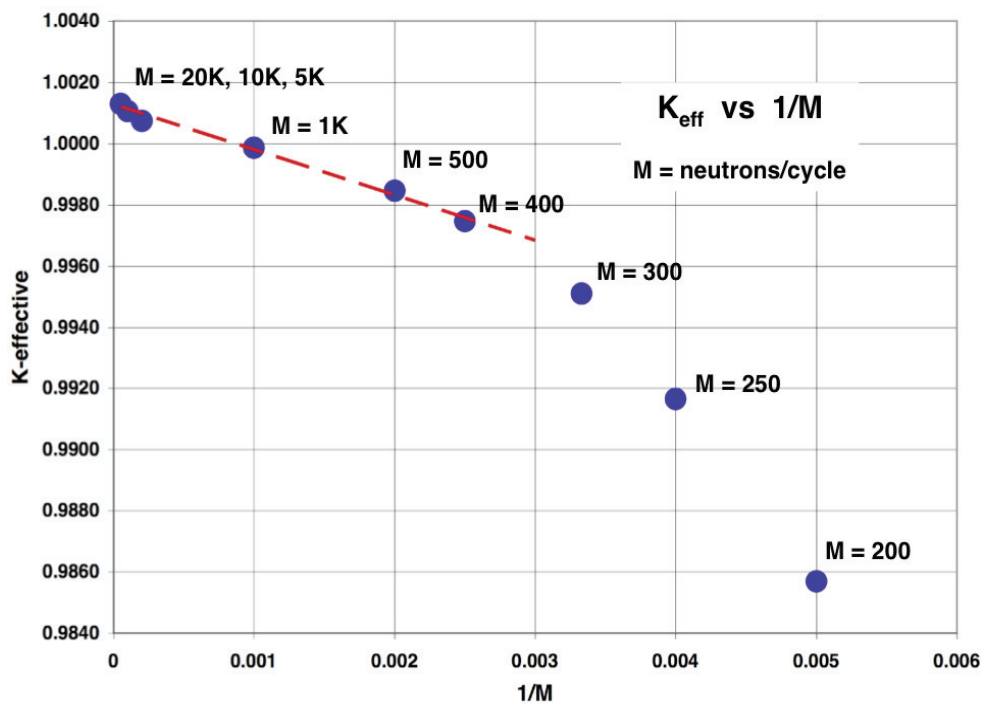

Fig. 1 K-effective of the World Problem, A - geometry model, B - convergence of $k_{e f f}$ and $H_{s r c}$ (y-axes for each plot: 0.8-1.2 for $k_{\text {eff, }}, 4-10$ for $\left.H_{s r c}\right), \mathrm{C}$ - distribution of $k_{\text {eff }}$ for replica calculations, D - $k_{\text {eff }}$ dependence on neutrons/cycle 
entropy of the fission source, vs. iteration cycle for various values of $M$, the number of neutrons/cycle. It can be seen that using larger $M$ makes it easier to diagnose convergence of both $k_{\text {eff }}$ and $H_{s r c}$, and that roughly 100 cycles are required to converge both $k_{\text {eff }}$ and the fission source distribution. Figure $1 \mathrm{C}$ shows the distribution of $k_{\text {eff }}$ values from several hundred independent replica calculations (i.e., identical calculations run with different random number seeds), for several different values of $M$. It can be seen that the spread in the $k_{\text {eff }}$ distribution is reduced for larger $M$. The shift in the peak values is due to reducing the renormalization bias in $k_{\text {eff. }}$. Figure 1D shows this more clearly, and demonstrates that the bias is inversely proportional to $M$.

In order to get accurate, reliable results for the K-effective of the World problem, it can be inferred from Fig. 1 that 10,000 or more neutrons/cycle should be used, and that 150 or more initial cycles should be discarded before beginning tallies.
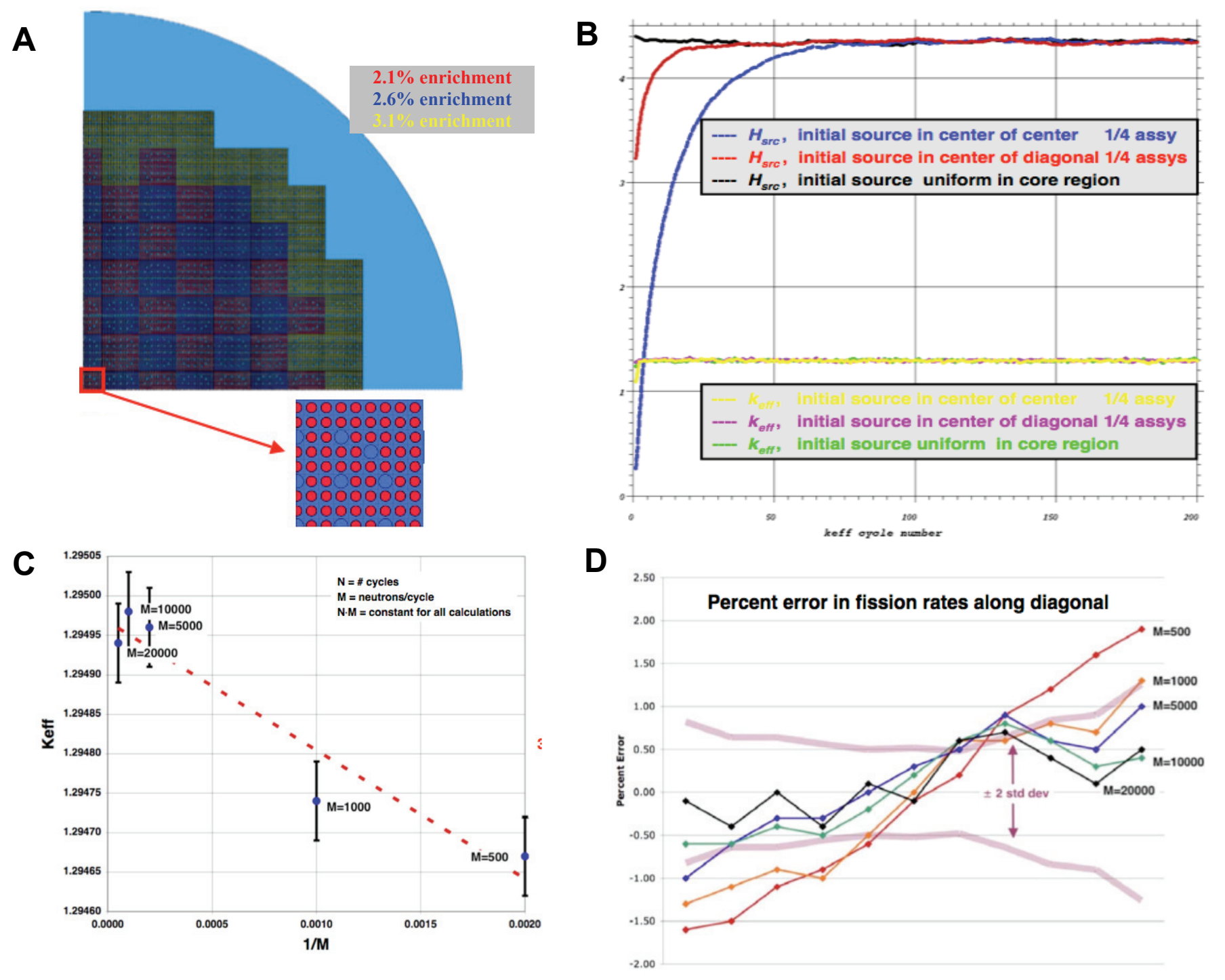

Fig. 2 Quarter-core PWR Problem, A - geometry model, B - convergence of $k_{\text {eff }}$ and $H_{\text {src }}, \mathrm{C}-k_{\text {eff }}$ dependence on

neutrons/cycle, $\mathrm{D}$ - bias in fission distribution along core diagonal for various $M$

\section{Quarter-Core PWR Problem}

Figure 2 provides results from MCNP5 calculations of a proposed by Nakagawa and Mori. ${ }^{11)}$ Figure $2 \mathrm{~B}$ shows the convergence behavior for $k_{\text {eff }}$ and $H_{s r c}$ for different choices of the initial guess for the fission distribution. Using a point source guess requires many cycles to converge to the fundamental mode; using a line source on the core diagonal is somewhat better; using a uniform fission source guess throughout the core region is the preferred initial guess, since it is nearly converged. It can also be clearly seen that $H_{s r c}$ take longer to converge than $k_{\text {eff. }}$. Figure 2C shows the dependence of $k_{\text {eff }}$ on $M$. Using 10,000 or more neutrons/cycle for this problem is sufficient to eliminate the $k_{\text {eff }}$ bias. Figure 2D shows the fission distribution along the core diagonal for various $M$. Clearly, the fission distribution is also biased when $M$ is chosen too small. Choosing 10,000 or more neutrons/cycle eliminates the bias in the source shape for this problem. 2D quarter-core model of a PWR similar to a benchmark 


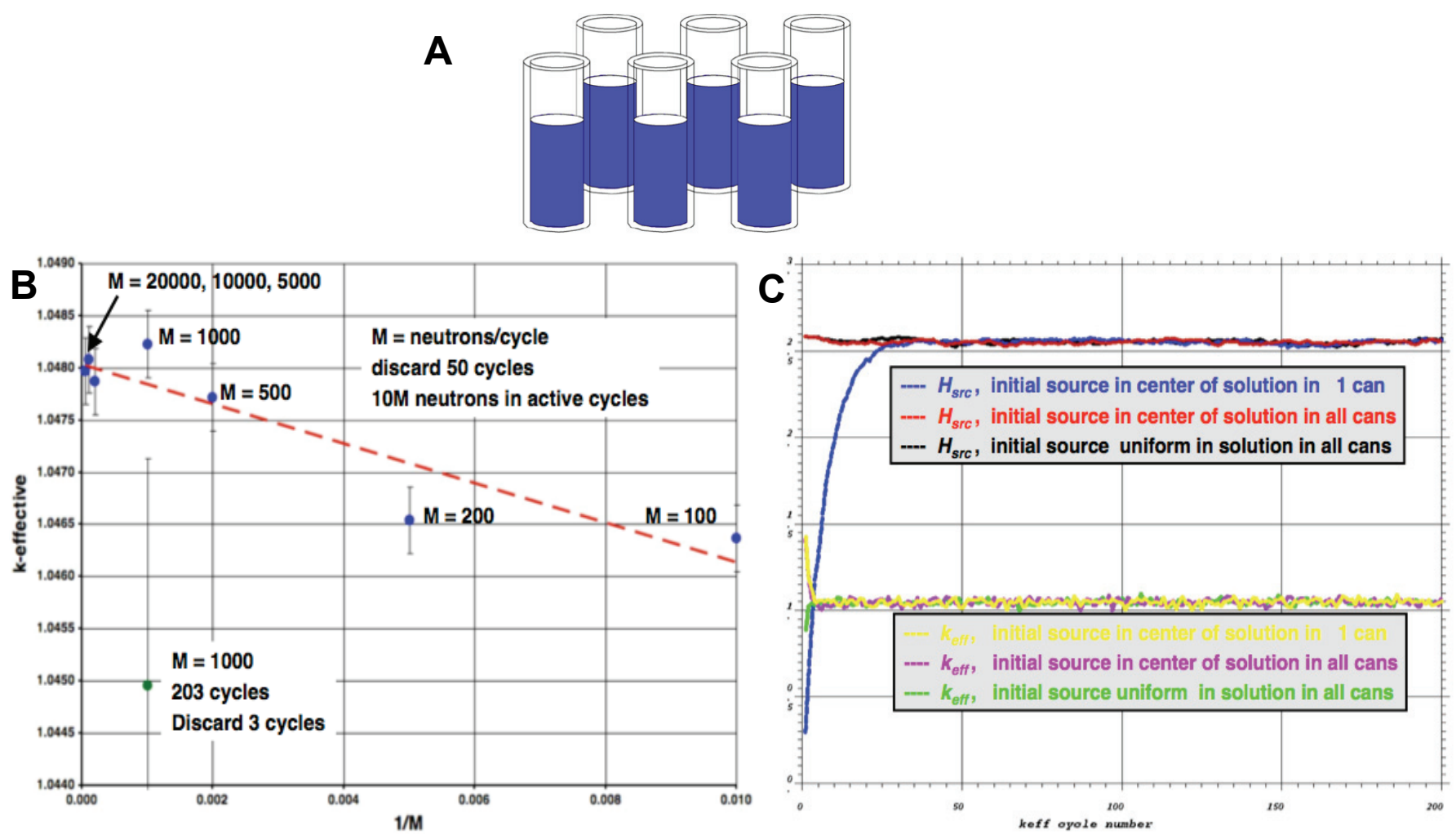

Fig. 3 Array of cans partially filled with plutonium nitrate solution, A - geometry model, B $-k_{\text {eff }}$ dependence on neutrons/cycle, $\mathrm{C}$ - convergence of $k_{\text {eff }}$ and $H_{s r c}$ for various $M$

While 10,000 or more neutrons/cycle is sufficient for this problem to prevent undersampling and to eliminate bias, even more neutrons/cycle will be required for 3D whole-core reactor problems. Experience has shown that 100,000 or even 1,000,000 neutrons/cycle should be used in large 3D problems to provide adequate Monte Carlo sampling and reliable local tallies. Fortunately, these large problems can readily be run using parallel computation, and the efficiency of parallel execution increases for larger $M$.

\section{Array of Cans Filled with Plutonium Nitrate Solution}

The $2 \times 3$ array of steel cans partially filled with plutonium nitrate solution is a simplified version of the problem described in Chapter 5 of the MCNP Criticality Primer. ${ }^{12}$ ) There are six stainless steel cylinders arranged in a $2 \times 3$ array with a $10 \mathrm{~cm}$ separation between cylinders. For simplicity, no external walls or features are included. Results are shown in Fig. 3.

Figure $3 \mathrm{~B}$ shows the computed values for $k_{\text {eff }}$ for the array of cans using 100, 200, 500, 1000, 5000, 10000, and 20000 neutrons/cycle. For this problem it can be seen that using 100 or 200 neutrons per cycle results in a bias of about 200 $\mathrm{pcm}$, and that using 1,000 or more neutrons per cycle effectively eliminates the bias in $k_{\text {eff. }}$ Also shown in Fig. $3 \mathrm{~B}$ is the $k_{\text {eff }}$ result (the green point) for the array of cans using 1,000 neutrons per cycle, but using an incorrect number of discarded cycles. In that run, only 3 cycles were discarded before beginning the $k_{\text {eff }}$ tallies, rather than 25 cycles for the other runs. The bias introduced by beginning the tallies before convergence is significant.
Figure 3C shows the convergence behavior of $k_{\text {eff }}$ and $H_{s r c}$ for different choices of the initial fission source guess. A single point source is a poor guess, leading to longer convergence; uniform points within all individual cans is better.

\section{Conclusions - Best Practices}

Based on both theory and numerical results, the following guidance and recommendations constitute best practices for Monte Carlo criticality calculations:

\section{Convergence}

For the initial source guess in a criticality calculation, choose a uniform distribution in all fissionable regions of the problem. If only a one or a few source points are used, more cycles will be needed to assure convergence.

To determine the number of cycles needed for convergence of the power iteration method, always make a trial run using $\sim 100$ cycles and a moderate number of neutrons per cycle (e.g., 1,000). Examine plots of both $k_{\text {eff }}$ and $H_{s r c}$ vs cycle to determine the number of cycles to be discarded before beginning tallies.

For applications where only $k_{\text {eff }}$ is sought, examine plots of $k_{\text {eff }}$ vs. cycle to determine the proper number of cycles to discard before beginning the $k_{\text {eff }}$ tally. For applications where local tallies are required (e.g., local reaction rates, foil measurements, dose fields, fission distributions, etc.) in addition to $k_{\text {eff, }}$, examine plots of both $k_{\text {eff }}$ vs. cycle and $H_{s r c}$ vs. cycle to determine the proper number of cycles to discard before beginning the tallies. Be sure that final production runs are 
made using at least that many discarded cycles; using fewer discarded cycles can bias the results.

\section{Bias in $\boldsymbol{k}_{\text {eff }}$ and Tallies}

It is recommended that 10,000 or more neutrons/cycle be used for all calculations. For large 3D reactor or storage vault problems, 100,000 or more neutrons/cycle is preferred. Problems should never be run using 10 s or 100 s of neutrons/cycle; that would introduce significant bias in both $k_{\text {eff }}$ and any local tallies.

\section{Bias in Uncertainties}

The uncertainties computed for $k_{\text {eff }}$ and reaction rate tally distributions exhibit a bias due to inter-cycle correlation effects that are neglected when performing the Monte Carlo code tallies. ${ }^{2,3,7)}$ The computed uncertainties are always smaller than the true uncertainties for a tally, regardless of the number of cycles run or the number of neutrons/cycle. The underprediction of uncertainties for local reaction rate tallies is often as large as factors of $3-5$, while the underprediction is generally small or negligible for uncertainties on $k_{\text {eff. }}$.

At present, there is no easy means of overcoming the underprediction bias in the computed uncertainties from Monte Carlo criticality calculations. While there is evidence that modifications to the iteration procedure, such as the superhistory method in $\mathrm{MONK}^{3)}$ and Wielandt's method under development for MCNP5, ${ }^{13)}$ can reduce or eliminate the underprediction bias in uncertainties, these methods are not available yet to general MCNP5 or SCALE/KENO users. A brute-force method for assessing the true uncertainties can be carried out: Make 25 or so independent Monte Carlo criticality calculations, discarding the uncertainties from the individual calculations, and compute the true uncertainties from the ensemble of results from the 25 runs.

\section{Summary}

With today's high-performance computers, it is straightforward to follow the above guidance when performing Monte Carlo criticality calculations. With improved understanding of the theory, new tools for diagnosing convergence (e.g., Shannon entropy of the fission distribution), and clear practical guidance for performing calculations, practitioners should have a greater degree of confidence than ever of obtaining correct results for Monte Carlo criticality calculations.

\section{Acknowledgment}

This work was supported by the US Department of Energy Nuclear Criticality Safety Program.

\section{References}

1) J. Lieberoth, "A Monte Carlo Technique to Solve the Static Eigenvalue Problem of the Boltzmann Transport Equation," Nukleonik, 11, 213 (1968).

2) E. M. Gelbard, R. E. Prael, "Monte carlo Work at Argonne National Laboratory," Proc. NEACRP Meeting of a Monte Carlo Study Group, ANL-75-2, Argonne National Laboratory, Argonne, IL (1974).

3) R. J. Brissenden, A. R. Garlick, "Biases in the Estimation of Keff and Its Error by Monte Carlo Methods," Ann. Nucl. Energy, 13, 2, 63-83 (1986).

4) G. E. Whitesides, "A Difficulty in Computing the k-effective of the World," Trans. Am. Nucl. Soc., 14[2], 680 (1971).

5) R. N. Blomquist et al., Source Convergence in Criticality Safety Analysis, Phase I: Results of Four Test Problems, OECD NEA No. 5431, OECD Nuclear Energy Agency (2006).

6) R. N. Blomquist et al., "NEA Expert Group on Source Convergence Phase II: Guidance for Criticality Calculations," 8th International International Conference on Criticality Safety, St. Petersburg, Russia, May 28 - June 1, 2007 (2007).

7) F. B. Brown, "A Review of Best Practices for Monte Carlo Criticality Calculations," ANS NCSD-2009, Richland, WA, Sept 13-17 (2009).

8) F. B. Brown, "On the Use of Shannon Entropy of the Fission Distribution for Assessing Convergence of Monte Carlo Critiicality Calculations," Proc. PHYSOR-2006, Vancouver, British Columbia, Canada (2006).

9) X-5 Monte Carlo Team, MCNP - A General N-Particle Transport Code, Version 5 - Volume I: Overview and Theory, LA-UR-03-1987, Los Alamos National Laboratory (LANL) (2003).

10) F. B. Brown et al., MCNP5-1.51 Release Notes, LA-UR-09-00384, Los Alamos National Laboratory (LANL) (2009).

11) M. Nakagawa, T. Mori, "Whole core calculations of power reactors by use of Monte Carlo method," J. Nuc. Sci. Techol., 30 [7], 692-701 (1993).

12) R. Brewer, Criticality Calculations with MCNP5: A Primer, LA-UR-09-00380, available at URL: mcnp.lanl.gov/publication/mcnp_publications.html (2009).

13) B. C. Kiedrowski, F. B. Brown, "Using Wielandt's Method to Eliminate Confidence Interval Underprediction Bias in MCNP5 Criticality Calculations," Trans. Am. Nuc. Soc., 99, 338-340 (2009). 